\title{
Lecciones de memoria. El recuerdo de 1971 en las protestas estudiantiles de 2011 en Colombia
}

\author{
Memory lessons. The memory of 1971 \\ in the student protests of 2011 in Colombia
}

Edwin Cruz Rodriguez

Candidato a doctor en Estudios Políticos y Relaciones Internacionales de la Universidad Nacional de Colombia. ecruzr@unal.edu.co

Artículo de investigación

Fecha de recepción: 25 de octubre de 2013 • Fecha de aprobación: 30 de noviembre de 2013

\section{RESUMEN}

Este trabajo muestra cómo la actualización de la memoria sobre la protesta estudiantil de 1971 sirvió, cuarenta años después, para afianzar la unidad y superar los sectarismos de las movilizaciones por el derecho a la educación en Colombia. En primer lugar, se reconstruyen los rasgos generales de ambos episodios, y luego se examinan las formas en que la memoria alrededor de lo ocurrido en 1971 operó para trazar una ruta al movimiento de 2011.

Palabras clave: memoria, protestas estudiantiles, Colombia, movimiento estudiantil.

\section{Abstract}

This paper shows how the actualization of memory about the 1971 student protest served, forty years later, to strengthen the unity and overcome the sectarianism of mobilizations for the right to education in Colombia. First, the general features of both episodes are reconstructed and then examines the ways in which memory about what happened in 1971 worked to define a route for movement of 2011. 
Keywords: Memory, student protests, Colombia, student movement.

\section{INTRODUCCIÓN}

Con el sugestivo titular Grito de amor y de protesta, una portada de la revista Semana, de noviembre de 2011, presenta a un joven estudiante abrazando a un policía antimotines en la Plaza de Bolívar de Bogotá. La imagen sirvió a los periodistas para hacer un contraste entre las formas de protesta -muchas veces violentas- que caracterizaron la gran movilización estudiantil de 1971 y las de 2011, cuyo contenido lúdico y pacífico ganó la admiración de la opinión pública. El texto de la revista asevera: "un movimiento estudiantil que hace apenas unos meses no existía logró convocar las manifestaciones más grandes desde 1971” (Semana, 2011).

No solo la prensa recurrió a la memoria de 1971. En la primera plenaria de la Mesa Amplia Nacional Estudiantil (Mane), en Bogotá el 20 y 21 de agosto de 2011, se decidió conformar un comité operativo, "cuya primera reunión se [llevaría] a cabo en Palmira, el 10 de septiembre, a propósito de los 40 ańos del "Programa Mínimo de Palmira” (Mane, 2011a). Más tarde la Mane confeccionaría su propio Programa Mínimo, a la altura de los retos de la educación superior en 2011.

Un componente importante de la subjetividad en las protestas estudiantiles de 2011 fue la memoria de las movilizaciones de 1971. En efecto, a la oposición frente a la propuesta de reforma de la Ley 30 de 1992, que regula el sistema de educación superior, se adicionó en dicha coyuntura la conmemoración de los 40 ańos de las movilizaciones que, hasta entonces, habían representado a un mismo tiempo los mayores triunfos, empezando por el logro del cogobierno universitario en algunas universidades durante pocos meses, y los más grandes fracasos en la historia del movimiento estudiantil. De manera explícita, o implícita, las huellas que las luchas de una generación en pleno Frente Nacional habían dejado se proyectaron sobre las organizaciones, los discursos, los proyectos y los repertorios de acción de una generación que llegaba a la mayoría de edad junto con la progresista Constitución de 1991 (Archila, 2012). 
Como lo evidenciaron los seminales estudios de Rivera (1986; 2010) acerca del papel de la memoria en los movimientos kataristas e indianistas en Bolivia, este es un factor fundamental para comprender aspectos como la elaboración de los discursos políticos, las estrategias y las formas organizativas de los movimientos sociales. La memoria, que involucra el recuerdo y el olvido no es inmutable y objetiva, sino un producto social en constante transformación (Archila, 2004, p. 16).

El pasado está en permanente construcción mediante los mecanismos de la memoria; hace parte de cómo las personas dan sentido a su mundo en el presente, elaboran su realidad social y se proyectan al futuro (Manero \& Soto, 2005, p. 173). Sostiene Jelin (2002, p. 18) que "el pasado que se rememora y se olvida es activado en un presente y en función de expectativas futuras". Existen coyunturas y procesos sociales como los rituales y lo mítico que activan distintos procesos de conmemoración.

Los movimientos estudiantiles colombianos actualizan y resignifican el pasado en forma constante, en sus prácticas cotidianas, mediante los "rituales de lucha" y las conmemoraciones. Estas prácticas recrean memorias de onda larga, como la conmemoración del día del "estudiante caído" que recuerda los asesinatos de Gonzalo Bravo Páez (8 de junio de 1929) y de Uriel Gutiérrez (9 de junio de 1954) (Aguilera, 2011, p. 184; Díaz, 2012). La marcha de los estudiantes de la Universidad Industrial de Santander hacia Bogotá, en julio de 1964, actualizó la memoria de la insurgencia anticolonial puesto que en su recorrido los estudiantes "se asimilaron con los Comuneros liderados por José Antonio Galán” (Acevedo \& González, 2011a, p. 265).

La memoria de acontecimientos más recientes, o de "onda corta", tiene cabida en las prácticas de los movimientos estudiantiles, como los procesos de represión de principios de los años ochenta o las protestas de 1971. Ahora bien, en las coyunturas de actualización de la memoria esta no solo se recrea a la luz de las circunstancias del presente, sino que el proceso de resignificación del pasado siempre está inmerso en una lucha política entre distintas maneras de recordar u olvidar, vale decir, entre distintas memorias (Jelin, 2002, p. 6; Delgado, 2008, p. 17). 
En dicha perspectiva, este trabajo estudia la actualización de la memoria de la protesta estudiantil de 1971 en las movilizaciones de $2011^{1}$. Se argumenta que las dinámicas de unidad en el movimiento estudiantil de 2011 atenuaron las disputas por la memoria y, en su lugar, le confirieron un papel aleccionador en el que el recuerdo de 1971 funcionó como un orientador de aquello que no se debía repetir y de lo que era necesario emular, particularmente en cuanto a la necesidad de interpelar la sociedad, superar el sectarismo y construir una organización unitaria o gremial.

En primer lugar, se reconstruyen en líneas gruesas ambos episodios de protesta estudiantil. En el segundo, se examinan las formas en las que la memoria de 1971 operó para el movimiento de 2011.

\section{LA PROTESTA ESTUDIANTIL EN 1971 y 2011}

\section{La experiencia ambigua de 1971}

La movilización estudiantil de 1971 hizo parte de los picos más altos de protesta durante el Frente Nacional (Archila, 2003, p. 142). Las movilizaciones en los primeros meses de ese año constituyeron "el mayor desafío de los sectores subalternos al régimen bipartidista” (p. 105).

El movimiento estudiantil de 1971 es un desenlace de un proceso de más largo aliento que se retrotrae hasta 1954 (Vargas, 1996, p. 61). Ese año tuvo lugar la masacre de estudiantes a manos de la dictadura rojista. Tres años más tarde, en 1957, el movimiento estudiantil pasó a convertirse en el héroe para las élites bipartidistas descontentas con el gobierno de Rojas, al participar activamente en su caída. Sin

1 La recolección de información para este análisis se basó en una revisión de la historiografía especializada en el movimiento estudiantil de 1971. Para reconstruir las protestas de 2011 se usaron técnicas de observación participante en acciones colectivas y eventos del movimiento estudiantil en ese ańo, así como un seguimiento de prensa (Revista Semana, El Tiempo, El Espectador) y la realización de 11 entrevistas semiestructuradas a "informantes clave" de distintos procesos organizativos, como son concebidas por Blee y Taylor (2002, pp. 105107). De esas entrevistas solo tres abordaron explícitamente al tema de la memoria y son las que se citan en las referencias. 
embargo, una vez instaurado el Frente Nacional, hacia 1961, el estudiantado empezó a ser visto como un sector proclive a la subversión. Tanto así que en 1965 se produjo una gran represión y la desarticulación de la organización estudiantil del momento. De acuerdo con Leal (1984, p. 158), a partir de 1969 el movimiento estudiantil pasó a ser considerado como un problema de orden público.

El presidente Misael Pastrana se posesionó en 1970 bajo estado de sitio, implantado luego del fraude electoral y se levantó en mayo pero fue restablecido el 19 de julio, un día antes de su posesión. Se trataba de un contexto muy complejo de crisis de legitimidad del Frente Nacional que abrió oportunidades políticas para distintos actores sociales y políticos, empezando por el movimiento campesino y el movimiento estudiantil (Múnera, 1998).

La reforma agraria y el establecimiento del estatuto de control de capitales en el marco del Grupo Andino, iniciativas del anterior gobierno de Lleras Restrepo, dividían a las élites bipartidistas. Buena parte de la Alianza Popular Nacional (Anapo), ante el fraude electoral, dio un giro a la izquierda y en varias ocasiones se articuló a los liberales en oposición al Frente Nacional. Para completar, la Asociación Nacional de Usuarios Campesinos (Anuc) emprendía un proceso de autonomía dirigido por diversas tendencias de izquierda que se expresaría en un ciclo de toma de tierras a comienzos de 1971 (Pécaut, 1989, p. 135).

El conflicto que propiciará el ciclo de protesta estudiantil en 1971 se originó muy temprano, en junio de 1970, en la Universidad del Valle. Allí los estudiantes de la División de Ciencias Sociales y Economía demandaron reformas al Consejo Superior Universitario (CSU) tratando de implantar el cogobierno universitario, mayor calidad académica y democratización el gobierno universitario, en particular la elección del decano (Acevedo \& González, 2011b, p. 224).

El problema radicaba en el vínculo que el decano tenía con la Fundación de Educación Superior (FES), que implementaba proyectos financiados por agencias norteamericanas, en el marco de la reforma universitaria contemplada en el Plan Básico, y que era visto por el estudiantado como una intervención imperialista (Archila, 2012, p. 82). 
A principios de 1971 el panorama para Pastrana era aún más complejo, pues a la crisis de legitimidad con que arrancó su mandato se adicionó la efervescencia social (Hernández, 2007, p. 38). Luis Carlos Galán fue nombrado Ministro de Educación. La Universidad del Valle, la Universidad Industrial de Santander y la Universidad Nacional habían recibido préstamos del Banco Interamericano de Desarrollo (BID) y permanecía candente la pugnacidad en torno a la reforma universitaria (Cote, 2009, p. 417).

Según Hernández (2007, pp. 39-40), todos los sectores estudiantiles concordaban en demandas como: expulsar los representantes de la Iglesia de los consejos superiores universitarios, el rechazo a la intromisión norteamericana en las políticas de educación superior mediante sus agencias financieras y las iniciativas comprendidas en el Plan Básico, mayor presupuesto para la universidad pública, y autonomía e investigación de acuerdo a las necesidades del país.

El 29 de enero de 1971 los estudiantes de la División de Ciencias Sociales de la Universidad del Valle entran en un paro de 24 horas. El 8 de febrero hay una huelga general y en la Universidad Nacional se inician protestas por el déficit presupuestal, problemática que también afectaba otras universidades (Cote, 2009, pp. 418-419). Por esa razón, el paro fue respaldado por estudiantes de otras universidades públicas y privadas en todo el país. El 26 de febrero se preparó una marcha nacional de solidaridad con la protesta en Cali que terminó con una cifra indeterminada de estudiantes muertos (entre 20 y 30). Este suceso, así como la represión y estigmatización de la marcha como "subversiva" por parte del gobierno y la declaración del estado de sitio, extendieron el movimiento en el ámbito nacional. Más adelante, el gobierno de Pastrana trató de apabullar la movilización planteando una reforma universitaria, que fue rechazada por los estudiantes e incluso por otros estamentos universitarios (Archila, 2012, p. 82).

De acuerdo con Le Bot (1979, pp. 106-107), el conflicto se desarrolló en tres períodos. Entre febrero y abril de 1971 la correlación de fuerzas fue favorable a los estudiantes, que presentaron un programa coherente de reforma, mientras el gobierno osciló entre promesas de reforma y represión. De mayo a septiembre la iniciativa fue del gobierno, que empieza a controlar las universidades recurriendo 
a la ocupación militar y depone el proyecto de reforma previamente planteado, el 29 de julio. Entre octubre de 1971 y mayo de 1972 tiene lugar la experiencia de cogobierno, que hace que los conflictos se prolonguen.

La iniciativa gubernamental, desde abril de 1971, no se explica solamente por la represión que implementó, sino también por las tensiones internas del movimiento estudiantil en relación con la reforma. Algunos sectores aceptaban negociar una reforma, mientras otros se inclinaban por agudizar las tensiones hasta llevar a la revolución. El sector favorable a una reforma, liderado por la Juventud Patriótica (Jupa) y representado por Marcelo Torres, fue escuchado por los rectores. El ministro Galán aceptó paliar el déficit presupuestario de la Universidad Nacional e implementar una reforma a los consejos superiores universitarios a fin de mejorar la representación estudiantil. Sin embargo, el cogobierno fue de poca duración, se desmontó a fines de ańo y se nombraron gobiernos universitarios autoritarios conocidos como "rectores policía” (Archila, 2012, p. 83).

A lo largo del año el movimiento desarrolló una gran articulación con seis encuentros estudiantiles, algunos en condiciones de clandestinidad. En uno de ellos se confeccionó el Programa Mínimo, ratificado por representantes de 30 universidades, con excepción de los representantes de la Universidad La Gran Colombia, en Palmira los días 3 y 4 de abril (Federación de estudiantes de la Universidad del Valle-FEUV-, 1973, p. 92). Sobre esa base se estableció el cogobierno en la Universidad Nacional y la de Antioquia, que rigió hasta el cambio de ministro, de Luis Carlos Galán a Juan Jacobo Muñoz, quien dictó decretos para suprimir los consejos superiores universitarios (Hernández, 2007, p. 46).

Por lo anterior, la memoria del movimiento de 1971 sería ambigua. Por una parte, se reivindicó la capacidad de generar unidad entre los distintos sectores del movimiento estudiantil a nivel nacional, generar protesta y formular propuestas. Pero, por otra parte, también se recordaría el fracaso de la experiencia del cogobierno en la raíz del cual estaban las disputas internas entre los sectores reformistas y revolucionarios, que además de minar la unidad y la capacidad de protesta, terminaron por alejar el movimiento de los problemas de la sociedad colombiana (Acevedo \& González, 2011b, p. 235). 


\section{El triunfo parcial de 2011}

En contraste, los actores del movimiento estudiantil en 2011 hicieron un balance positivo en términos de una "victoria parcial". Entre marzo y noviembre de ese año, la sociedad colombiana fue sacudida por la protesta. El movimiento estudiantil desarrolló grandes movilizaciones para oponerse al proyecto de reforma a la Ley 30 de 1992, que regula el sistema de educación superior, consiguió articular a su lucha diversos sectores, planteó un Programa Mínimo, y logró que el gobierno retirara su propuesta y se dispusiera a construir una reforma concertada ${ }^{2}$.

Desde fines de 2010 se empezó a hablar de la reforma a la Ley 30. Aún no se sabía si el gobierno mantendría el proyecto radicado por la administración Uribe, limitado a lo financiero. El 10 de marzo, el presidente Santos presentó públicamente una ambiciosa propuesta que, según el gobierno, recogía experiencias internacionales exitosas en el aumento de cobertura y calidad de la educación superior. Se centraba en la atracción de la inversión privada en las universidades públicas y el establecimiento de instituciones de educación superior (IES) con ánimo de lucro. El debate se concentró en la posibilidad de privatización de la educación, en las perversiones de la inversión privada para la autonomía universitaria, y en si el ánimo de lucro era una alternativa idónea para aumentar calidad y cobertura ${ }^{3}$.

Los críticos — rectores de universidades públicas y privadas, voceros de organizaciones estudiantiles, congresistas, entre otros- argumentaban que la reforma dejaba de lado la calidad para centrarse en la cobertura, iba en contra de la autonomía universitaria y de los criterios de pertinencia para las necesidades del país, y concebía la educación como mercancía en vez de derecho.

La ministra de educación, María Fernanda Campo, afirmó que los recursos públicos no eran suficientes y por eso era necesario vincular al sector privado, el proyecto de ley daba más recursos a la educación y las alianzas público-privadas no equivalían a privatizar ni vulneraban la autonomía universitaria. Además, se mostró afanada

2 Para una detallada reconstrucción del proceso ver Cruz (2012).

3 Para un análisis ponderado de la propuesta de reforma gubernamental ver: Miñana y Rodríguez (2011). 
por dar trámite legislativo a la reforma, argumentando que la administración tenía tiempos distintos a la academia, y descalificó a sus adversarios, sobre todo a los voceros estudiantiles, aseverando que desconocían la propuesta. La interlocución se planteó como “pedagogía”, no como construcción colectiva.

El movimiento estudiantil, que no estaba activo desde las protestas contra el Plan de Nacional de Desarrollo de la segunda administración Uribe (2007), emprendió un proceso de unidad sin precedentes en su historia reciente. El Encuentro Nacional Estudiantil del 19 y 20 de marzo acordó la creación de la Mane para construir la unidad programática, organizativa y de movilización, y definió una agenda de protestas nacionales. Era un momento de crisis, administrativa y financiera, de gran parte de las universidades públicas y algunas privadas, y de aumento en los niveles de protesta estudiantil a nivel local y regional. El descontento en las regiones empezó a articularse en las movilizaciones nacionales del 7 de abril, y 12 y 17 de mayo.

El éxito de las movilizaciones atrajo la atención pública y ubicó el problema en la agenda gubernamental. El 23 de agosto, el gobierno anunció el retiro del ánimo de lucro. Esta decisión se produjo luego de una reunión de la Mesa de Unidad Nacional, que reúne los partidos de la coalición de gobierno, entre ellos el Partido Verde, en el que había opiniones divididas. Fue una victoria para los opositores y mostró que la unanimidad de la coalición podía fracturarse. Sin embargo, para el movimiento estudiantil el ánimo de lucro solo había desaparecido formalmente, pues se mantenía la confusa figura de las "universidades mixtas". Sus voceros articulaban la crítica a la mercantilización de la educación con la de los tratados de libre comercio, demandaban el retiro de la propuesta y tomar el tiempo necesario para construir una reforma concertada.

El 1 de octubre, el Comité Operativo de la Mane decidió que entraría en paro nacional universitario una vez el proyecto fuera radicado en el Congreso para trámite legislativo. Así sucedió dos días después, el 3 de octubre.

El 12 de octubre las universidades públicas entraron en paro, apoyadas por un buen número de estudiantes de universidades privadas. Empero, los argumentos 
del gobierno no cambiaron. A un gran despliegue mediático, con propagandas de televisión en horarios triple A que llamaban a los ciudadanos a no dejarse "engañar" por los opositores a la reforma, se sumó la deslegitimación del paro nacional universitario, resaltando sus altos costos, y el llamado para que la discusión se hiciera en el Congreso, no en las calles.

La protesta se extendió con jornadas de movilización el 7 y 12 de octubre, con cerca de 20.000 y 40.000 participantes, respectivamente. Acciones de proporción similar tuvieron lugar el 19 y 26 de octubre, y el 3 y 10 de noviembre, aprovechando las audiencias públicas sobre la reforma, citadas en el Congreso. El gobierno tenía de su lado a la Mesa de Unidad Nacional, los rectores habían moderado sus críticas luego del retiro del ánimo de lucro y la entrada en paro, pero el movimiento ganaba el pulso en la opinión pública.

En las audiencias, los voceros estudiantiles demostraron un gran conocimiento de la política educativa y dejaron la sensación de triunfo de sus argumentos. Pero el gobierno no cedía, el presidente instaba a los estudiantes a cesar el paro y afirmaba que no encontraba argumentos para protestar.

El Comité Operativo de la Mane, el 5 de noviembre, hizo un balance de 31 universidades públicas en paro, la mayoría de las regionales del Servicio Nacional de Aprendizaje (SENA) y 25 universidades privadas sumadas a la protesta. La movilización no tenía precedentes y el proceso organizativo de la Mane se había replicado en las regiones. Se decidió no participar en las discusiones del proyecto de ley en la Comisión Sexta de la Cámara de Representantes, considerando que el Congreso no era un espacio democrático por tener mayorías de la coalición gubernamental, y realizar una jornada internacional de movilización en defensa de la educación como derecho el 24 de noviembre.

No obstante, el 9 de noviembre, el gobierno tomó una decisión no anticipada por el movimiento. Tras varias peticiones de congresistas de los partidos Liberal y de la U, en la Mesa de Unidad Nacional, el presidente anunció que retiraría el proyecto si los estudiantes levantaban el paro. Ello evidenció cierta fractura en la coalición gubernamental. Empero, era también una estrategia para intervenir en la "Toma 
de Bogotá” programada para el día siguiente. Aun así, el movimiento persistió en la protesta. La Mane emitió un comunicado afirmando que no levantaba el paro hasta que no se cumplieran sus demandas, y convocó a una plenaria de emergencia el sábado 12 de noviembre.

Al día siguiente se llevó a cabo la "Toma de Bogotá", con alrededor de 120.000 personas de todas las regiones y una congregación en la Plaza de Bolívar. La movilización fue tan contundente que el presidente anunció el retiro del proyecto de ley y aseguró que el gobierno estaría abierto a discutir la reforma. En esa decisión pesaba el daño que su imagen empezaba a sufrir como consecuencia de las movilizaciones y las diferencias en el interior de su coalición. Tras un álgido debate y el compromiso del gobierno de proveer las garantías para construir una reforma concertada, la Mane inició la suspensión del paro el 16 de noviembre.

En suma, la experiencia ambigua de las movilizaciones de 1971, puso en jaque al Frente Nacional pero fue incapaz de consolidar la reforma universitaria que instauraba el cogobierno universitario. En contraste, el balance inmediato que se hizo de las protestas en 2011 fue de una victoria relativa, en tanto que se consiguió que el gobierno retirara su propuesta de reforma y se orientara a construir una propuesta concertada. Sin embargo, como se verá enseguida, tal contraste no resulta obvio en las representaciones de los acontecimientos de 1971 que los estudiantes de 2011 evocan, en la medida en que no tienen por objeto ser fieles a los hechos del pasado sino darles un sentido en función de las necesidades del presente y los proyectos del futuro.

\section{LaS HUEllaS DE 1971 EN 2011}

Para Jelin (2002, p. 30), el pasado deja huellas que se expresan en conductas rutinarias, entramados simbólicos o marcas materiales como los monumentos y el espacio, que requieren un "trabajo de la memoria", una agencia subjetiva que las actualice y las dote de sentido en función de las circunstancias presentes.

En 2011 convergieron una memoria autobiográfica que se expresó en la representación que los protagonistas de 1971 hicieron de su experiencia, y una memoria histórica, 
la de aquellos estudiantes que no experimentaron los acontecimientos de 1971 pero accedieron a ese pasado por otros medios (Halbwachs, 2004, p. 54).

Las huellas de las protestas estudiantiles de 1971 llegaron a la generación que protestó en 2011 por distintas vías, como la transmisión del conocimiento intergeneracional propio de la militancia en las organizaciones estudiantiles, las conmemoraciones en distintos medios de prensa e incluso la participación de algunos de los protagonistas de entonces como invitados en eventos del movimiento estudiantil. Por ejemplo, para el Encuentro Nacional Programático de la Mane, a realizarse el 12 de noviembre de 2011, se planeó la asistencia de un docente que hubiese participado en el movimiento de 1971(Mane, 2011b).

Una fuente destacada fue el acceso a la historiografía especializada en los movimientos estudiantiles. Así, si bien la disciplina de la historia y la memoria tienen grandes diferencias en la forma como relacionan el pasado, el presente y el futuro (Archila, 2004), en ciertas coyunturas la memoria, como la actualización y resignificación del pasado en función del presente, bebe de la historia. Por tal razón, es conveniente seguir el recuerdo de 1971 de cerca con la reconstrucción que de esa coyuntura ha hecho la historiografía.

La forma predominante que adopta la memoria de 1971 en 2011 es la de la lección, esto es, de aquello que hay que evitar repetir y de aquello que es deseable emular. Las lecciones aparecen en términos de los triunfos y los fracasos del movimiento de 1971, y también están presentes en la historiografía. A grandes rasgos, se refieren a la necesidad de interpelar la sociedad, superar el sectarismo y construir una organización unitaria o gremial.

\section{Interpelar la sociedad}

Entre las cuestiones a imitar se encuentra, en primer lugar, la necesidad de una reflexión crítica sobre las condiciones sociales del país por parte del estudiantado, que sea capaz de "hablarle" a la sociedad. Al reconstruir parte del movimiento en los años setenta, Santiago Castro (s.f.), afirma que aunque el movimiento se caracterizó por su fugacidad, constituyó un sector muy importante de oposición al Frente Nacional y 
llevó a los estudiantes, incluidos los de universidades de elite, a realizar una reflexión crítica sobre la educación, la universidad y el país.

En el mismo sentido, para Pardo y Urrego (2003, p. 5), el movimiento tuvo "un triunfo sin antecedentes en la historia nacional", en la medida en que consiguió elaborar una propuesta de gobierno universitario y contrarrestar el influjo de organismos internacionales en la política educativa. No resulta extraño entonces que, apelando a la memoria y a los aciertos y errores que esta permite identificar, un estudiante, entonces vocero de la Mane, afirmara:

El movimiento estudiantil que ha visto Colombia en este último tiempo es un movimiento sincero que le habla al país como piensa, que actúa como siente. Es el mismo movimiento estudiantil heredero de la rabia, del genocidio... El mismo inscrito en las memorias de una historia, por muchos olvidada, de asesinatos impunes, de represión legitimada, de indiferencia cómplice... El movimiento estudiantil no nació este año, su expresión reciente es una semilla decantada de la experiencia, los aciertos y errores de muchos tiempos: el tiempo de la desidia, el tiempo del entusiasmo, el tiempo de la indignación, el tiempo del miedo, el tiempo del horror, los tiempos comunes de nuestro movimiento social (Rivera, 2011, pp. 54-55).

En un sentido similar, un colectivo de estudiantes de la Universidad Nacional llamado Memoria y palabra, realiza un balance del movimiento en 2011 que resalta justamente su capacidad de interlocución con la sociedad. La forma como se articula la memoria de 1971 con las luchas de 2011 es bastante particular, pues su evocación del movimiento de 1971 se centra en el momento de la unidad y la capacidad de convocar el debate público, sin mencionar las disputas internas que dieron al traste con la experiencia, lo que permite realizar cierta equivalencia entre los dos procesos:

Creemos que al igual que el movimiento estudiantil de 1971 esta movilización ganó fortaleza al consolidar un programa mínimo, que ha centrado el debate y unificado la lucha estudiantil para exigir el cumplimiento de unos objetivos básicos... De manera similar en este último periodo de movilización estudiantil hemos ganado una opinión pública favorable, la sociedad entera quiere escuchar 
a los voceros de la Mane y no podemos perder dicha interlocución. Entonces surge la pregunta ¿Qué tiene hoy que decirle el movimiento estudiantil a la huelga de la USO, al paro petrolero, a los trabajadores precarizados de todos los sectores, a los que luchan por la tierra, a las víctimas, etc.? Pero la pregunta va en doble vía ¿Qué aprende y cómo interlocuta el resto del movimiento social con los estudiantes? (Memoria y palabra, 2011).

Ello permite ver hasta qué punto la memoria es siempre selectiva, no tiene como criterio guía el proveer una información ajustada a los "hechos", sino que en los procesos de rememoración existe una relación compleja entre recuerdo y olvido en función de las necesidades de dar sentido al pasado en función del presente y el futuro.

De la mano de la necesidad de interpelar a la sociedad, de "hablarle al país", sobre sus problemas, se ubica también una cierta capacidad de agencia que provee el acto de rememoración y que se evidencia particularmente en el deseo de estar a la altura de los acontecimientos cuyas cuatro décadas se conmemoran en 2011. Por una parte, al recordar los acontecimientos de 1971 en pleno auge de la protesta de 2011, el entonces líder de la Juventud Patriótica, Marcelo Torres, recapitula las medidas represivas, el estado de sitio, el cierre y la militarización de las universidades, pero al mismo tiempo sentencia: "ganamos el forcejeo" (Linares, 2011). En forma involuntaria fija un horizonte para el movimiento de 2011 que enfatiza en la victoria a pesar del difícil contexto en que tuvo lugar la protesta de 1971.

Por otra parte, en otro reportaje uno de los voceros de la Mane explica el letargo de 40 años del movimiento estudiantil señalando: "hay tres razones que llevaron a esta dispersión: la represión y persecución del Estado, el sectarismo de las organizaciones estudiantiles y la falta de memoria histórica que nos llevaba a caminar sobre los mismos errores del pasado" (Gutiérrez, 2011), y al ser interrogado por las razones de la resurrección del movimiento estudiantil, enfatiza en la necesidad del cambio: "los jóvenes y los estudiantes de Colombia volvimos a tomar conciencia de que otro mundo no solo es posible, sino necesario". Con ello alude a una visión en cierto sentido cíclica del tiempo histórico ("volvimos a tomar conciencia"), que tácitamente ubica el pasado en el presente mediante el acto de recordar, y resalta la capacidad 
del movimiento para aprender de los errores del pasado, así como la voluntad de transformación provista por el hecho de ponderar la conducta de los estudiantes de la actualidad en función de las pautas establecidas por el recuerdo de 1971.

\section{Superar el sectarismo}

Como se acaba de ver, la memoria juega un papel aleccionador en el movimiento estudiantil, en la medida, señala lo que se debe aprender de las experiencias del pasado. En este sentido, también lo que se ha concebido como falencias o errores del movimiento de 1971 operan en la memoria del presente. Entre ellas se enmarca la necesidad de superar el sectarismo en el interior del movimiento.

Para varios autores, las enconadas disputas ideológicas en las distintas tendencias del movimiento estudiantil de 1971 están en la raíz de la explicación del fracaso del cogobierno, puesto que encerraron al movimiento sobre sí mismo y sus disputas internas, llevándolo a aislarse de los problemas de la gente, impidiéndole interpelar a las personas fuera de las universidades e incluso privándolo de su propia audiencia estudiantil (Le Bot, 1979, pp. 94-95; Castro-Gómez, s.f., p. 95; Archila, 2012, pp. 83-84; Beltrán, 2002, p. 161). En efecto, pese al esfuerzo de unidad que había llevado a la adopción del Programa Mínimo, en el interior del movimiento subsistían varias tendencias con propuestas políticas e interpretaciones del contexto divergentes.

De acuerdo a un análisis de la época (aa. vv., 1971, p. 46), las diferencias entre las organizaciones estudiantiles se estructuraban en función de la respuesta que tenían para tres problemas: la naturaleza de la universidad en el marco del capitalismo y el papel que en ese contexto deberían tener las luchas estudiantiles; la lectura de la coyuntura a nivel nacional de cara al proceso revolucionario; y las alianzas y repertorios de acción a implementar.

La Juventud Comunista (Juco) tenía una posición a favor de la reforma, entendiendo que era necesario conseguir cambios graduales que permitieran ir transformando paulatinamente la situación social. La Juventud Patriótica (Jupa), agrupación juvenil del Movimiento Obrero Independiente Revolucionario (MOIR), tenía una lectura similar y veía la democratización del gobierno universitario como un paso 
importante hacia la gran transformación de la sociedad. Algo similar ocurría con el Centro de Estudios Sociales o Frente de Estudios Sociales, también de tendencia maoísta (Archila, 2012, pp. 83-84).

Sin embargo, otros núcleos organizativos tenían respuestas distintas para el mismo problema: a grandes rasgos, pensaban que la universidad era un organismo encargado de reproducir el orden social existente y, en consecuencia, era necesario primero cambiar ese orden social para luego sí construir otro tipo de universidad. Esta era la línea de pensamiento de lo que en la época se denominó el "campo socialista”, donde convergían distintas organizaciones de tendencia trotskista y entre las cuales se encontraba la Tendencia Socialista, con bastante arraigo en la Universidad del Valle (Cote, 2009, p. 442). Alrededor de esos polos se ubicaban otras organizaciones (aa. vv., 1971, p. 42; Le Bot, 1979, p. 110). Lo cierto es que una vez que la Jupa venció en las elecciones para el CSU de la Universidad Nacional, en noviembre de 1971, la polarización llevó a un punto de no retorno que acabó por minar la capacidad de convocatoria del movimiento (Cote, 2009, p. 437).

Por consiguiente, es comprensible que parte de la memoria sobre 1971 esté dada por evitar el "error" del sectarismo. Así por ejemplo, entre las enseñanzas que un estudiante, integrante de la organización Proceso Nacional Identidad Estudiantil, extraía para la Mane se encontraba:

No repetir errores históricos: podríamos mencionar en este punto que los intentos de construir una plataforma de lucha nacional del estudiantado, por diferentes razones han tendido a convertirse en una organización política particular o diluirse con el tiempo... Hoy se le plantea a la Mane un reto bien grande y es el no caer en manos de una organización específica (Pérez, 2012, p. 4).

También un vocero de la Mane sostuvo que la unidad en las protestas de 2011 en buena parte había sido posible por una transformación y un abandono del sectarismo entre las organizaciones estudiantiles, en comparación con los movimientos de los años setenta: 
Lo primero [que ha cambiado en el movimiento estudiantil] es la unidad, el movimiento estudiantil se ha podido unir... Ha cambiado eso y es que no es un movimiento sectario, es decir, nosotros y nosotras, en la posibilidad que hoy tenemos de fortalecer este movimiento estudiantil hemos tenido un marco para poder discutir con procesos estudiantiles de antaño, que tuvieron fuerza en los setenta, que se han acercado a plantearnos y a decirnos: vea un maoísta no se podía ver con un 'trosco' pues porque se encendían, o sea, era literal, era a puńos. Mientras que hoy vemos por ejemplo que usted ve a pelados de la FEU andando con los de la OCE, ve gente de la FUN andando con pelados de REO. Ahí hay un proceso unitario en el movimiento estudiantil... Sí, tenemos diferencias, pero nos ponemos de acuerdo en que Esta es la prioridad" (entrevista 1).

\section{Construir una organización gremial}

El sectarismo se expresaría en buena medida en la imposibilidad de construir una organización gremial de los estudiantes a nivel nacional. En 1957, luego de la participación de los estudiantes en la caída de Rojas Pinilla, se fundó la Unión Nacional de Estudiantes Colombianos (Unec), que se proclamaba gremial e independiente de los partidos y el Estado, aunque reivindicaba la consolidación de la democracia y una reforma orgánica de la universidad (Ruiz, 2002, pp. 68-69). Sin embargo, un par de años más tarde la experiencia de unidad había sucumbido ante la toma de posición política de varias tendencias en su interior.

En noviembre de 1963, en el Tercer Congreso Nacional de Estudiantes realizado en Bogotá, se constituyó la Federación Universitaria Nacional (FUN), también como una organización de tipo "esencialmente corporativo", donde confluían varias organizaciones de carácter nacional en función de la reivindicación de la autonomía universitaria: la Unec, la Juco, las Juventudes del Movimiento Revolucionario Liberal (JMRL), la Confederación de Estudiantes Universitarios de Colombia (CEUC, que agrupaba estudiantes de universidades privadas, Javeriana y Andes, principalmente) y el Movimiento Obrero Estudiantil Campesino (MOEC) (Uribe, 1998, pp. 519-520). 
De acuerdo con Vargas (1996, p. 53), la FUN fue una cristalización de los congresos de unidad desarrollados ese ańo y se constituyó en una organización que superó las disputas entre el bipartidismo y las vertientes del MRL y la Anapo, más exactamente representó los ideales de la "nueva izquierda" y su oposición al Frente Nacional. Por todo ello, hasta la actualidad se recuerda como la principal experiencia de unidad en la historia del movimiento estudiantil colombiano.

En su interior también se produjeron fracturas entre las tendencias reformistas y las de otros grupos, como las que se expresaron en su Segundo Congreso de octubre de 1964 (aa. vv., 1971, p. 34). Sin embargo, la principal causa para la desaparición de la FUN se ubica en la represión a la que fue sometida en octubre de 1966 por el gobierno de Lleras Restrepo, luego de que fuera abucheado en el campus de la Universidad Nacional, cuando se disponía a inaugurar un edificio del Instituto Colombiano Agropecuario en compañía de Nelson Rockefeller, quien había financiado la iniciativa. Era la segunda vez que a Lleras Restrepo le sucedía algo similar — la primera fue el 6 de noviembre de 1964_, pero esta vez tenía la calidad de presidente de la república. Como respuesta, determinó la ocupación militar del campus, la disolución de la FUN y del CSU de la Universidad Nacional, el arresto y el procesamiento mediante consejos verbales de guerra a los estudiantes responsables (alrededor de 100), además de retirar a los representantes estudiantiles del CSU (Le Bot, 1979, p. 96; Ruiz, 2002, p. 209).

Lo significativo es que luego de la FUN nunca se volvió a presentar una experiencia de unidad de esas proporciones. De hecho, la fragmentación del movimiento en 1971 y, por consiguiente, su derrota al momento de consolidar el cogobierno se han explicado por "la ausencia de una organización gremial de carácter nacional", dado que las organizaciones estudiantiles dependen de estructuras políticas que definen su línea de actuación y terminan por perjudicar sus demandas sectoriales (Archila, 2012, pp. 83-84).

En efecto, la memoria del fracaso en el intento por construir una organización unitaria es predominante a la altura de 2011, cuando se llegó a explicar ese fracaso por la ausencia de una organización gremial del estudiantado colombiano. Como recuerda el integrante de Identidad Estudiantil: 
Las luchas y los esfuerzos por construir una organización unitaria de los y las estudiantes colombianas. En este punto son varias las experiencias a resaltar, por ejemplo el empeño que se le puso a la creación de la Federación Nacional de Estudiantes en el año de 1922 inspirados en el proceso de Córdoba, Argentina o la creación en 1935 de la Federación de Estudiantes Colombianos (FEC), luego a estos dos intentos fallidos y posterior a la revolución cubana se intenta de nuevo organizar el estudiantado colombiano con un proceso que se llamó Unión Nacional de Estudiantes Colombianos que por diversas situaciones no salió adelante. Ya para el año de 1963 y de la mano de Camilo Torres Restrepo y Jaime Arenas, entre otros líderes estudiantiles, se crea la Federación Universitaria Nacional (Pérez, 2012, p. 4).

De ahí que para los voceros de la Mane, integrantes de organizaciones estudiantiles de carácter nacional, asumieran como principal reto en 2011 el de construir la unidad en un amplio proceso organizativo (Rivera, 2011, p. 55). De hecho, la memoria del déficit organizativo estuvo presente durante todo el proceso de formación de la Mane, hasta tal punto que para una de sus voceras en 2011, se trata de un proceso organizativo que había llegado tarde.

El movimiento estudiantil usualmente llega a puntos de madurez y retrocede, y bueno creo que estamos en un punto de madurez... La Mane tiene mucho por aportar, desafortunadamente la Mane nace muy tarde, porque otra historia estaríamos contando si se hubiera formado hace dos años (entrevista 2).

Se trata entonces de proceso en construcción resultado del aprendizaje de experiencias anteriores de unidad. Para otro estudiante y vocero de la Mane, esta experiencia es la síntesis de lo que denomina la "historia viva", que técnicamente podemos hacer equivalente al concepto de memoria, presente en el movimiento estudiantil.

[Para entender cómo se llega a la Mane] hay que tener en cuenta varios elementos, un poco la historia viva que tiene el movimiento estudiantil, que es de décadas y décadas atrás, que siempre ha intentado sintetizarse en experiencias de procesos organizativos. Entonces pues ahí hay varios intentos anteriores a la Mane como puede ser la Federación Universitaria Nacional, 
que alcanzó a consolidar el proceso gremial... Como un proceso gremial, que pretendía aglutinar los estudiantes... Y eso es lo que viene a ser la Mane, viene a ser síntesis de todo eso (entrevista 3).

La más reciente de esas experiencias de unidad que se "sintetizan" en la Mane fue la Coordinadora Nacional de Estudiantes Universitarios (CNEU). La CNEU se creó por iniciativa de las organizaciones de carácter nacional del momento, en el Congreso de Estudiantes de Bucaramanga, en 2003, para hacer frente a unos decretos del gobierno sobre acreditación y presupuesto y, entre otras cosas, desarrolló el paro de abril de 2005. Tenía como objetivo una organización gremial con unidad en lo organizativo, lo programático y la movilización, y se reunió hasta dos veces por semestre. Aunque podrían participar organizaciones locales y regionales y estudiantes no organizados, no se planteó como un espacio amplio sino de interlocución entre organizaciones. Luego del paro se produjeron desacuerdos entre ellas que terminarían por acabar con el proceso. Su última reunión fue en octubre de 2006 cuando las diferencias fueron inconciliables.

Desde 2007 se realizaron Encuentros Nacionales Estudiantiles Universitarios (ENEU), para coordinar movilizaciones coyunturales, por ejemplo, contra el Plan Nacional de Desarrollo de Uribe, en 2007, que obligaba a las universidades públicas a hacerse cargo de su pasivo pensional. Allí solo participaban las organizaciones estudiantiles y no tenían perspectivas en lo programático y lo organizativo. A fines de 2009 se empieza a discutir la necesidad de un espacio de unidad, que llevó al Encuentro de Manizales, el 14 y 15 de abril de 2010, para enfrentar la reforma a los artículos 86 y 87 de la Ley 30 propuesta por el gobierno Uribe. Finalmente, el Encuentro de marzo de 2011 decide crear la Mane para enfrentar la reforma a la Ley 30. Mesa amplia implica articular al estudiantado, no solo universitario, más allá de las organizaciones e idearios, mayoritariamente de izquierda, y avanzar en la unidad programática y organizativa.

El recuerdo de la dificultad para construir una organización unitaria del estudiantado es muy bien retratado por el colectivo Memoria y Palabra (2011) al posicionar históricamente la experiencia de la Mane. 


\begin{abstract}
Vemos en el actual proceso de la Mane un retorno a la unidad del movimiento estudiantil que se había gestado en la Federación de Estudiantes Colombianos tras la masacre del 8 y 9 de junio del 1954. La FEC estuvo compuesta por estudiantes de todas las tendencias políticas. También nos recuerda el proceso de los sesentas con una verdadera Federación Universitaria Nacional, cuya principal fortaleza fue tener expresiones locales de procesos estudiantiles como la Asociación Universitaria de Estudiantes de Santander (AUDESA)... La movilización estudiantil de 1971 propuso el cogobierno en las universidades públicas (administración conjunta de gobierno, estudiantes, profesores y trabajadores de los centros educativos)... No matemos las luchas estudiantiles en discusiones desgastadas, apostémosle a la unidad, o en simplemente recordar muertos. La memoria no debería ser para enterrar a nuestros muertos sino para revivirlos, una memoria insepulta. Una memoria que está adolorida y lastimada pero que, a partir de la experiencia camina y construye colectivamente.
\end{abstract}

En menos de cuatro meses la Mane se constituyó como una estructura de coordinación para avanzar hacia la unidad del estudiantado. En este proceso tuvo que lidiar con el cuestionamiento a los mecanismos de toma de decisiones en las asambleas, la emergencia de un discurso antiorganización que reivindica los estudiantes no organizados o de organizaciones locales, la discusión sobre las vocerías nacionales y la articulación con procesos regionales. Sin embargo, incluso los denominados "estudiantes independientes", críticos del manejo que las organizaciones estudiantiles con más experiencia daban a la Mane, no dudaron en apelar al recuerdo de 1971 para fortalecer el proceso unitario:

En 1971 los universitarios colombianos dentro de un proceso de movilización presentaron un programa mínimo que aglomeraba de manera estructural los principios de autonomía, presupuesto y política académica dentro de la universidad pública. Dicha lucha permitió que docentes y estudiantes hicieran parte de los organismos de decisión de las instituciones. Tal como hace cuatro décadas, los estudiantes presentamos una inconformidad con políticas gubernamentales que enfocan la educación superior a la simple y desdichada dinámica del mercado... La Mesa Amplia Nacional Estudiantil es el resultado de años de intentos gremiales, donde la unidad resultaba ser una 
premisa compleja y siempre las discrepancias distanciaron a las organizaciones estudiantiles... Dentro de la Mane, existen discrepancias notorias entre los diferentes sectores que la conforman. Los estudiantes independientes (que no pertenecemos a ninguna organización) sentimos que hemos sido discriminados al momento de elegir voceros, algunos grupos han acaparado la atención política con partidos y movimientos externos a la universidades, lo que choca con los otros, que consideramos la Mane como el escenario propio de la crítica, sin necesidad de torpedearla con intereses ajenos a ella. Los miembros de la Mane deben entender que el discurso y el debate es pluralista. Se notan los juegos de poderes al interior de las vocerías y el ánimo de figuración personalista de algunos sectores. Esta crítica surge desde adentro y es un llamado a todos, por dejar de lado tan nefastas prácticas asociadas más a procesos electorales que a procesos reales de construcción política (Parra, 2012).

\section{Corolario}

Este artículo examinó la forma en que se actualizó la memoria del movimiento estudiantil de 1971 en las protestas estudiantiles de 2011 en Colombia. El ejercicio de la memoria está ligado a la necesidad de vincular el pasado para darle un sentido de acuerdo a las necesidades del presente y los proyectos del futuro. Por consiguiente, se trata de formas de representación del pasado sustancialmente distintas de aquellas a las que aspira a elaborar la disciplina de la historia, en la medida en que la rememoración no siempre ni necesariamente tiene por objetivo establecer la veracidad de los hechos.

En este caso particular, las movilizaciones de 1971 se recordaron a fin de identificar aquellos factores que, a manera de "lecciones" históricas, había que emular o impedir que se repitieran. De esa manera, los distintos recuerdos fueron revestidos, en forma preponderante aunque no única, con el barniz de la "lección histórica".

Para los distintos protagonistas el pasado, actualizado bajo la forma del recuerdo, adoptó significado en función principalmente de lo que de él podría aprenderse así como de los retos que una situación similar, como la movilización estudiantil de 1971, establecía para el movimiento estudiantil en 2011. Por lo tanto, la búsqueda 
de "enseñanzas" o lecciones y el establecimiento de analogías con el presente guiaron las interpretaciones del pasado.

Así, si bien la resignificación del pasado que se opera mediante la memoria está atravesada por disputas políticas que permiten identificar distintas interpretaciones y sentidos de los acontecimientos, en este caso el carácter unitario de las protestas de 2011, que convergieron en la construcción de la Mane, opacó las disputas que pudieron presentarse entre distintos sectores del movimiento por la interpretación de los hechos y el significado de 1971 .

De manera tal que para el movimiento estudiantil de 2011 la memoria de 1971 adquirió sobre todo un carácter aleccionador, puesto que a partir de la rememoración de los acontecimientos, distintos actores del movimiento se orientaron a señalar la necesidad de superar de alguna manera los límites y "errores" que, en sus distintas interpretaciones, se cometieron en aquel momento o por otra parte, de emular aquellos "aciertos" que pudieron presentarse.

Ello permite comprender, finalmente, por qué la evocación de 1971 apareció siempre que se quiso resaltar la necesidad de interpelar a la sociedad, superar los sectarismos y construir una organización estudiantil unitaria.

En fin, aunque las movilizaciones de 2011 fueron cerradas con un diagnóstico, por parte de sus protagonistas, según el cual el retiro del proyecto de reforma educativa por parte del gobierno constituía una "victoria parcial" del movimiento, y en este sentido se estableció un contraste con lo que en ciertas memorias se percibía como el fracaso del cogobierno en 1971, lo más probable es que en el futuro, a la luz de los acontecimientos, las necesidades y sentidos de otro presente, ambos acontecimientos adopten otra significación. 


\section{REFERENCIAS}

Acevedo, Á. y González, D. (2011a). Protesta y movilización estudiantil, 1964. Memoria de una marcha en la Universidad Industrial de Santander. Anuario Colombiano de Historia Social y de la Cultura, 38(2), pp. 255-276.

(2011b). Movilización y protesta estudiantil en Colombia (1971). Una lectura desde la organización gremial por el cogobierno universitario y la memoria de protagonistas y testigos". Anuario de Historia Regional y de las Fronteras, 16, pp. 221-242.

Aguilera, A. (2011). Subjetividades politicas y movimientos sociales en defensa de la universidad pública: Colombia-México. Tesis para optar al título de Doctora en Estudios latinoamericanos. Universidad Nacional Autónoma de México.

Archila, M. (2003). Idas y venidas, vueltas y revueltas. Protestas sociales en Colombia 19581990. Bogotá: ICANH-CINEP.

(2004). La historia hoy: ¿memoria o pasado silenciado?. Historia y Sociedad, 10, pp. 15-33.

(2012). El movimiento estudiantil en Colombia. Una mirada histórica. OSAL, 31, pp. 71-103.

Beltrán, W. (2002). Del dogmatismo católico al dogmatismo de izquierda. El ambiente político en la Universidad Nacional en los años 60 y 70. Revista Colombiana de Sociología, 7 (2), pp. 155-178.

Blee, K., y Taylor, V. (2002). Semi-Structured Interviewing in Social Movement Research. En B. Klandermans y S. Suzanne (eds.), Methods of social movement research (pp. $92-$ 117). Minneapolis-London: University of Minnesota Press.

Castro-Gómez, S. (s.f.) El movimiento Cataluña. Recuperado de http://www.goethe.de/ges/ pro/ori68/es3051198.htm 
Cote, J. (2009). El movimiento estudiantil de 1971. En aa. vv. Una historia inconclusa. Izquierdas politicas y sociales en Colombia (pp. 413-462). Bogotá: Cinep.

Cruz, E. (2012). La MANE y el paro nacional universitario de 2011 en Colombia. Revista Ciencia Política, 14, pp. 140-193.

Delgado, C. (2008). Aportes de la teoría de la memoria colectiva al análisis político. Memoria y sociedad, 12 (24), pp. 115-124.

Díaz, A. (2012). El 8 de junio y las disputas por la memoria, 1929-1954. Historia y Sociedad, 22, pp. 157-189.

Federación de Estudiantes de la Universidad del Valle. (1973). Desarrollo político del movimiento estudiantil. Bogotá: Herrera Hermanos Impresores.

Gutiérrez Torres, C. (2011). "La resurrección del movimiento estudiantil". http://www.elespectador.com/impreso/vivir/articulo-310381-resurreccion-del-movimiento-estudiantil (9 de noviembre de 2011).

Halbwachs, M. (2004). La memoria colectiva. Zaragoza: Prensas Universitarias de Zaragoza.

Hernández, I. (2007). El programa mínimo de los estudiantes colombianos. Movimiento estudiantil universitario de 1971 por la universidad. Todo un país. Revista Historia de la educación colombiana, 10, pp. 29-57.

Jelin, E. (2002). Los trabajos de la memoria. Madrid: Siglo XXI.

Le Bot, Y. (1979). Educación e ideología en Colombia. Medellín: La Carreta.

Leal, F. (1984). La participación política de la juventud universitaria como expresión de clase. En M. Cárdenas y A. Díaz (eds.), Juventud y política en Colombia (pp. 155-203). Bogotá: FESCOL-Instituto SER. 
Linares Gómez, A. (2011). "Los estudiantes ganaron su primer pulso y lograron frenar la reforma”. http://www.eltiempo.com/politica/reforma-a-la-educacion-_10756109-4 (12 de noviembre de 2011).

Mesa Amplia Nacional Estudiantil, MANE. (2011a). “Declaración de la Mesa Amplia Nacional Estudiantil”. http://www.ocecolombia.com/2011/09/declaracion-de-la-mesaamplia-nacional.html (6 de septiembre de 2011)

Mesa Amplia Nacional Estudiantil, MANE. (2011b). "Conclusiones del Comité Operativo. 05 de noviembre de 2011”. http://funcomisionesmodep.org/index.php?option=com_co ntent\&view=article\&id=105: conclusiones-comite-operativo-05-de-noviembre-de2011\&catid=34:nacionales\&Itemid=37 (10 de noviembre de 2011).

Manero, R. y Soto M. (2005). Memoria colectiva y procesos sociales. Enseñanza e investigación en psicología, 10 (1), pp. 171-189.

Memoria y palabra. (2011). "Sobre el movimiento estudiantil y su historia". http://memoriaypalabra.blogspot.com/search?updated-max=2011-12-12T08:12:00-08:00\& maxresults $=7 \&$ start $=28 \&$ by-date $=$ false $(17$ de noviembre de 2011$)$.

Miñana, C. \& Rodríguez, J. (2011). Calidad, evaluación y acreditación en la educación superior: a propósito de un proyecto de reforma en Colombia. Pensamiento Jurídico, 31, pp. $155-185$.

Múnera L. (1998). Rupturas y continuidades. Poder y movimiento popular en Colombia 19681988. Bogotá: Cerec-Iepri-Universidad Nacional de Colombia.

Pardo, M. y Urrego, M. (2003). El movimiento estudiantil de 1971 en Colombia. Ponencia presentada por los autores en el Primer Congreso Internacional sobre Historia de las Universidades de América y Europa, realizado del 10 al 12 de julio de 2003 en la Universidad de Córdoba, Argentina. 
Parra, L. (2012). "La Antimane. Un retroceso en el movimiento estudiantil”. http://comunidad.semana.com/t5/Participe/La-AntiMANE-un-retroceso-del-movimiento-estudiantil/idi-p/15019 (6 de mayo de 2012)

Pécaut, D. (1989). Crónica de dos décadas de politica colombiana. Bogotá: Siglo XXI.

Pérez, S. (2012). La Mane: tensiones y retos en la construcción del movimiento estudiantil colombiano. Diálogos de derecho y política, 10 (4), pp. 1-9.

Rivera, S. (1986). Oprimidos pero no vencidos. Luchas del campesinado aymara y quechua de Bolivia 1900-1980. Ginebra: UNRISD.

. (2010). Ch'ixinakax utxiwa: una reflexión sobre prácticas y discursos descolonizadores. Buenos Aires: Retazos-Tinta Limón.

Rivera, J. (2011). Desafíos: hacia un movimiento estudiantil para la transformación y la propuesta. Revista Izquierda, 18, pp. 54-61.

Ruiz, M. (2002). Sueños y realidades. Procesos de organización estudiantil 1954-1966. Bogotá: Universidad Nacional de Colombia.

Semana (2011). "Grito de amor y de protesta”. http://www.semana.com/nacion/grito-amorprotesta/167389-3.aspx (12 de noviembre de 2011).

Uribe, M. (1998). Universidad de Antioquia: historia y presencia. Medellín: Universidad de Antioquia.

Vargas, L. (1996). Expresiones politicas del movimiento estudiantil AUDESA, 1960-1980. Bucaramanga: UIS.

aa. vv. (1971). Crisis universitaria colombiana 1971. Itinerario y documentos. Medellín: Ediciones El Tigre de Papel, 1971. 


\section{Entrevistas}

1. Integrante del Sindicato Estudiantil y vocero de la Mane, Bogotá, noviembre 17 de 2011.

2. Integrante de Conciencia Crítica y vocera de la Mane, Bogotá, noviembre 16 de 2011.

3. Integrante de Rebeldía Estudiantil Organizada y vocero de la Mane, Bogotá, noviembre 23 de 2011. 